\title{
Data-driven Business Model Innovation for 6G
}

\author{
Sriganesh K. Rao \\ Calligo Technologies Pvt Ltd, Bangalore, India \\ E-mail: Sriganesh_rao@hotmail.com \\ Received 18 July 2021; Accepted 07 November 2021; \\ Publication 16 December 2021
}

\begin{abstract}
Futuristic disruptive technologies like 6G, could further transform the way we live and work, beyond what was enabled by $5 \mathrm{G}$. The expected features could enable massive high-speed and low-latency connectivity across diverse devices giving rise to new applications enabling instant availability of data related to Smart Cities, Industries 4.0, Intelligent Transport Systems, Utilities, Healthcare, Security, Media \& Entertainment, etc. They will further make it possible to scale current real-time data collection strategies, adding more devices without the need for wired connections or fear of interference. Data is considered as the new oil due to tremendous possibilities it can bring in the business world through predictive and prescriptive analytics. This gives rise to the importance of data-driven business model innovation to provide actionable insights to the business owners, helping them to develop sustainable business models. Organizations using big data and analytics within their innovation process are more likely to remain competitive and sustainable. This paper discusses the importance of harnessing "Real-time Data" to create value, the challenges in harnessing value from data especially "dark data," the role of "Data-driven Business Model Innovation" in creating
\end{abstract}

Journal of ICT Standardization, Vol. 9_3, 405-426.

doi: 10.13052/jicts2245-800X.935

(C) 2021 River Publishers 
sustainable business and Intelligent Transport System Data-driven Business Model Innovation as an example.

Keywords: $6 \mathrm{G}, 5 \mathrm{G}$, business model innovation, data-driven business model innovation, data analytics, smart cities, intelligent transport systems.

\section{Introduction}

Business models describe the rationale of how an organization creates, delivers, and captures value. Evolving consumption patterns, advent of social media, and emerging opportunities in new sectors fueled by disruptive technologies, would create a need to innovate new business models. Business and Government leaders will confront change on many fronts - in the way business organize themselves, on how jobs are defined, how we use technology to interact with each other and the rest of the world, etc. Business leaders and policymakers need to consider their potential before they begin to exert their disruptive powers in the economy and society and create new business models to fully monetize and harness the features enabled by these technologies [1].

Data is considered as the new oil due to the tremendous possibilities it can bring in the business world through predictive and prescriptive analytics. Data is considered as an asset for organizations now. There will always be an on-going demand for more higher speeds and data both from business and consumers, impacting new domains and giving rise to new use cases. This will pave the way for the need for technologies "beyond 5G" going forward into the next decade. Futuristic disruptive technologies beyond 5G, termed as $6 \mathrm{G}$, could further transform the way we live and work, beyond what was enabled by $5 \mathrm{G}$. It is expected to provide features beyond $5 \mathrm{G}$, like (i) Furtherenhanced Mobile Broadband (FeMBB) with speeds 10 times faster than 5G, (ii) Advanced ultra-Reliable Low Latency Communications (AuRLLC) of $<1 \mathrm{~ms}$, and (iii) Enhanced massive Machine Type Communications (EmMTC) capable of supporting trillions of machines.

Further, the expectations from such technologies integrating communications, navigation, sensing and services, are to enable: (i) Human Bond communications allowing more holistic sensory information exchange utilizing all five senses - optical, aural, olfaction, gustation, and tactile; (ii) mind-to-mind communications or telepathy, (iii) $5 \mathrm{G}$ to satellite connectivity, etc. This could provide seamless provision of services everywhere including remote areas, on-board ships, aircrafts (in-flight services), moving trains, etc., and could enable ability for remote interactions on an emotional level. 
The above-described features could result in far more data-intensive applications giving rise to the need for harnessing "real-time" data to create value. However, there are challenges in deriving insights from "dark data" and limitations of traditional data analytics platforms and absence of datadriven organization culture. However, these challenges can be overcome by using the right type of tools and processes.

Organizations can become successful due to their business model innovation (BMI) rather than their product innovation. Organizations can disrupt their markets or create new markets by using innovative business models designed around internal or external data collection and performing advanced data analytics to provide an unmet customer need. Big data analytics is the driving force of Marketing today. Data-driven marketing is crucial to success in a hypercompetitive global economy. Organizations using big data and analytics within their innovation process are more likely to remain competitive and sustainable.

Intelligent transport systems including connected vehicles, autonomous vehicles, and UAVs offer tremendous data-derived opportunities which are explored further in detail; as an example.

\section{Importance of Harnessing "Real-time" Data to Create Value}

"Data Age" reports that the global datasphere will grow from 45 zettabytes in 2019 to 175 zettabytes by 2025 and nearly $30 \%$ of the world's data will need real-time processing. By 2025, around 6 billion people would be interacting with data every day. This would mean that about $75 \%$ of world's population will be interacting with data daily [2]. 6G technologies giving rise to new applications across multiple domains, would result in further data explosion.

Example:

- Connected vehicles, autonomous vehicles, UAVs.

- Smart cities with city-wide sensor network.

- Factories of the future.

- Security and surveillance.

- Utilities.

- Healthcare, etc.

IoT devices and sensors capable of real-time information collection, coupled with advanced analytics technology has made reliance on real-time data, practical. These data must be accessed in near real-time and processed instantaneously to derive maximum benefits of the data. 
Let us examine one of the above domains - Connected Vehicles which are equipped with hundreds of sensors, integrated with a great number of smart technologies. Connected vehicles communicate with the driver, other vehicles, roadside infrastructure, and the cloud. They collect immense amounts of internal and surrounding data. Sources of data in a connected vehicle are: [1]

- Electrical Control Units which control internal sensors and gives vehicle health data.

- External data like:

- Environment parameters - weather, temperature.

- Data about pedestrians, other vehicles, and road conditions.

- Shared data with other nearby vehicles.

- Data from third parties through cloud connectivity like traffic/weather data, etc.

Self-driving cars are expected to generate and consume around 40 terabytes of data for every eight hours of driving and would be generating about as much data as about 3000 people [25].

Vehicle data creates value by: [1]

- Data is valuable in elevating the driver experience. Improves driver experience by acting as a real-world sensor that surrounding vehicles can leverage to determine real-time traffic and weather conditions.

- Data exchanged between vehicles on the road, can reduce potential crashes and improve traffic flows, all of which contribute to enhancing the driver experience.

- Vehicle manufactures can derive value from vehicle data because the vehicle is the source generating the data that other companies and thirdparty developers need to build new applications that ultimately impact the driver experience.

- Vehicle makers can monetize both the data stream by allowing access to specific streams of anonymized real-time data to third party developers, as well as the actual application like real-time traffic route guidance, through royalties charged to the third parties.

Apart from the vehicle manufacturers and owners, vehicle data could also find its use in the following:

- Traffic department to improve traffic systems and prevent traffic congestions. 
- Weather and road condition survey throughout different regions.

- Disaster management and emergency service providers.

- Third party vehicle ride sharing providers.

Each of the above use cases can create value either by generating revenue, reducing costs, or enhancing safety and security.

Many research companies have estimated the business opportunity value from Connected Vehicle data. Harbor Research expects this value to be $\$ 47$ billion by 2023 , out of which $\$ 8.2$ billion would be from basic systems software and services having applications like location, routine diagnostics, and monitoring, the rest would be due to advanced value-added software and services having applications that include vehicle performance, contextualized in-vehicle experiences, and V2X (vehicle-to-infrastructure, vehicle-tovehicle, etc) [3]. McKinsey \& Company estimates the overall revenue pool from vehicle data monetization at a global scale to USD 450-750 billion by 2030 [4].

While 5G will make Internet of Things (IoT) possible, 6G would speed it up. 6G will deliver speeds 1000 times faster than $5 \mathrm{G}$, enable 1 Tbps peak data rate and have a latency of five times lower than $5 \mathrm{G}$, which can make almost instantaneous transmission of massive data possible. It is expected to have a much higher improvement in energy efficiency compared to 5G. $6 \mathrm{G}$ would enable network management away from cloud to the "edge" so that the response time is reduced. 6G would enable much higher quality video across different verticals. This could include stadiums, shopping malls, etc., which would result in huge increase in amounts of data used and generated by the consumers and systems. Content can be generated, transmitted, and shared irrespective of place and time. This would enable new data-driven business opportunities across various verticals. This would enable new use cases using augmented reality and virtual reality applications providing immersive, interactive customer experience resulting in generation of huge amounts of data which needs to be harnessed in real-time or near real-time.

The Media \& Entertainment industry will be greatly impacted by the way content can be utilized. The quality of the content alone will not ensure the success of this industry. Success also depends on how easily consumers can access the content and how they are able to interact with it. Companies will have to tap into the consumers behaviors, preferences, locations, spend abilities and patterns, etc. Companies will have to find ways to tap such data and analyze them to be able to ensure their business success. 
Combination of big data, analytics, and AI has led to the transformation of existing services and creation of many new data-intensive services. It is critical that organizations harness the power of technology and data into an effective strategy, to remain competitive and create significant value. Gartner global CIO survey, conducted in 31 industries across 41 countries, concluded that Big Data \& Analytics as the most disruptive force in enterprise technology [5].

6G will make it possible to scale current real-time data collection strategies even further, adding more devices without the need for wired connections or fear of interference. This would enable businesses to scale up their real-time data strategies, innovating data-driven business models to ensure businesses to remain competitive and sustainable.

\section{Challenges in Harnessing Value From Data and Overcoming Them}

Organizations and people tend to take decisions based on biases and beliefs if they do not base their decisions of data. Data replaces beliefs and provides competitive advantages.

Every day 2.5 quintillion bytes of data are created. Ninety percent of the world's data today has been created in the last two years alone. Increased quantity of data allows for more qualitative approach. Big data includes both structured and unstructured data including text, sensor data, audio, video, and $\log$ files. Ninety percent of data generated is unstructured data that computers cannot easily interpret. This includes tweets, photos, etc. [6]. The top big data challenge, as per Gartner's CIO survey, is "determining how to get value from big data" and integrating big data technology with existing infrastructure [7].

According to "Data Age 2025" [21], companies will be storing more than 100 trillion gigabytes of data by 2025 . These data would be coming from various sources in different forms and hence harnessing these data into actionable insights would be the biggest challenge for any organization. It is estimated that only $37 \%$ of companies claim to have been data-driven [22].

In its July 2020 Global Survey report, IDC projected that the enterprise data will grow at $42.2 \%$ annually in the next two years and that only $32 \%$ of data available to enterprise is put to work while the remaining $68 \%$ is not utilized. The biggest factor impacting growth in amount of data going forward is attributed to increased use of advanced data analytics, followed by increased use of IoT devices gathering data automatically [24]. 
Lot of valuable information is hidden as "dark data" which is not easily visible or accessible. Organizations collect large amount of data in the normal course of functioning but may not be able to utilize them for reasons including either being unaware of them or limitation of the analytics platforms used. Such data is called as "dark data." Such data may result to lost opportunities if the important insights are not harnessed. Such organizations may lose their competitive advantage against their competitors who implement platforms to analyse and process dark data. Also, unanalysed data could create security or legal issues, if not handled correctly.

IBM estimates that roughly $90 \%$ of data generated by sensors and machines never gets used [23]. The growing flow of machine and sensor data generated by IoT, and the massive stores of raw data found in the unexplored depths of the deep web, all comprise dark data.

Dark data can give greater insights to reveal trends, patterns, and relationships that are missed during normal business intelligence and analytics activities. Dark data analysis could give better understanding of the customers' requirements and helps in reframing business insights. However, dark data analysis is very challenging for organizations.

Harnessing the unstructured data can enable discovery of hidden insights, which could be extremely useful to organizations. For example, social media data can help detecting customers/public perception of a product. If found negative perception, a pattern may be detected which may help in root-cause analysis, thereby improving the product/service quality and sustaining the business. Customer service can also utilize the social profile of the customer to better understand and deal with the customer.

The major challenges for advertisers today are understanding the scale, delivery, and feedback measurements, which can be overcome with datadriven business model innovation.

There is an availability of huge amount of data and information in every vertical and all spheres of life, which is enabled by IoT, 5G \& beyond technologies. However, there are challenges in effective harnessing of these data to create sustainable value. The challenges include:

- Lack of human and system abilities to properly ingest, analyze, and manage these data and information.

- Lack of effective data-driven strategy. The organization processes and current business models may not be data-driven.

- Data protection regulations introduced in various countries.

- Evaluating and defining the role and influence of AI - lack of explainable AI. 
- Lack of the right resources and skills to bring data, analyze it, get actionable insights.

- Data ingestion to connect well-structured data to semi-structured/ unstructured data that is not easily understood.

- Data volume would be huge, and the companies will have to be able to access it in real-time, analyze, and act on that instantly. Systems should be capable of handling any amount of data very quickly.

- Cost of implementation of harnessing value from data.

Forbes survey revealed that the major challenges to adopting analytics were that the analytics insights were not well integrated into the business process and there was lack of people skills in implementing them [8].

Sensors can be installed anywhere easily but effective utilization of the data they produce could be a challenge as a certain pattern needs to be derived from the data. Digitalization and data bring with it, new business models and new opportunities to create value and generate revenues. Furthermore, gathering, storing, and processing data is associated with costs that companies need to weigh against the potential income it can bring. A process of value creation is needed, where data is made useful by processing and combining it in the right ways.

Dark data can be harnessed by identifying the useful data from large amount of unstructured data by using the right tools and applying data pattern algorithms. The value of a particular amount of data can be identified to determine areas of its usefulness and risks if not utilized. Such tasks cannot be done manually and needs the right kind of tools to harness actionable insights. Tools utilizing artificial intelligence, machine learning, computer vision, pattern recognition, etc. will have to used.

Traditional and legacy data analytics platforms are too complex to build, very slow, have limitations on handling dark data, and are not scalable. Thus, they are unable to harness data effectively to get actionable insights when they must handle the type and volume of data enabled by $6 \mathrm{G}$ technologies. The above limitation can be overcome by using the right kind of data analytics platform which can do the predictive and prescriptive analytics of structured, unstructured, and semi-structured data.

The data analytics platform should enable better data capture, interpretation, analysis, and visualization data from any form and from any type of sources. Traditional platforms depend on humans for data preparation, analysis, and visualization. It should provide AI at the data ingestion, data preparation, and data visualization. It should be able to move from descriptive analytics through predictive analytics to prescriptive analytics. 
Data analytics platform should include the following features:

- Support for text analytics, NLP, image \& video processing using computer vision algorithms.

- Data ingestion mechanisms for streaming, logs, bulk, micro-batches.

- Support for structured, semi-structured, and unstructured data.

- Features for data cleansing and visualization.

- Ability to have AI across all layers - at data ingestion, data cleansing, and data visualization. Traditional platforms have AI at data cleansing layers only.

- Optimized for high-performance to ensure scalability. The platform should support high-performance compute and high-performance storage.

- Built-in data anonymization for personal data protection.

- Data encryption for enhanced security.

- Should be edge deployable. Decisions must be taken at the Edge or at the place where the action happens. This ensures real-time analytics.

- Traditional platforms may be proprietary resulting in vendor lock-in. The data analytics platform should support global standards.

- Cost-effective and should not increase the cost of harnessing data than the value generated by it.

It is very important for organizations to embrace a data-driven culture and strategy and implement data-driven business model innovation. Data analytics mainly involved analysing structured data. With the understanding of the importance of "Dark data", harnessing value out of dark data gives organizations a competitive edge.

\section{Role of Data-driven Business Model in Driving Sustainable Innovation}

In Section 2, we have seen the importance of harnessing "real-time" data to create value.

Data-driven business models innovation should fully unlock the commercial potential of their data, making better use of data across the board, including info sourced from customers and telemetry from smart devices, biometrics/voice data, purchase behavior, transaction data, etc.

Data-driven business model innovation can be used in Business to Consumer (B2C), Business to Business (B2B), Business to Business to Consumer (B2B2C), and Government to Citizen (G2Ci) [9]. There is rapid urbanization 


\section{S. K. Rao}

going around in the world. Effective collection and utilization of data is the key to the success of Smart Cities [10].

Irrespective of their size, businesses can look at ways to monetize their own big data to capture new revenues. This gives rise to need for them to create data-driven business models. Well-known companies have become successful, due to their data-driven business model innovation rather than their product innovation [11]. Such companies can disrupt their markets or create new markets by using innovative business models designed with the insights provided through data analytics from internal or external data being harnessed, with result of providing unmet customer needs. Examples Google, Facebook, Amazon, Netflix, Uber, Airbnb, Zara, Flipkart, OLA, etc [1].

Organizations using big data and analytics within their innovation process are $36 \%$ more likely to remain competitive and sustainable [12].

MIT Research showed that data-driven businesses have an output and productivity that is $5-6 \%$ higher than similar organizations who are not utilizing data-driven processes [13].

Data-driven marketing is increasingly adopted and claimed by $65 \%$ of marketing executives as crucial to success. Twenty percent of marketing expense is used for data-driven advertising campaigns. Big data analytics is widely adopted in marketing today and is considered as its driving force [14].

Some examples - Vehicle manufacturers can change their BMs from product-oriented strategy of selling more vehicles, to a customer- and serviceoriented strategy like "custom design for individual" enhancing customer experience.

Data-driven business model innovation helps in creating business continuity plans ensuring sustainable business in face of disruption. Business models should involve faster adoption of new technologies, more investments in technologies, new ways of working, and data-driven technologies will be the key in this.

Business models will have to adapt to changing social attitudes, customer behaviors, and demands, as power has shifted to the consumers in the digital age where they can compare products, services, prices, and reviews, and buy in an instant from any part of the world. Customers' expectations are increasing, and preference is for buying or working with socially responsible organizations. Business models must cater for different generations of customers, as the aspirations and brand loyalty vary vastly. Gen $\mathrm{Z}$ have lower brand loyalty compared to earlier generations. Such businesses will have to use data-driven insights as a way of meeting rising customer expectations. 
Business models would have to include sustainability to environment also by including waste reduction and lowering energy consumption in their activities. Sustainable BMs have the following advantages:

- Customer's preference to buy or work with sustainable organizations.

- Cost savings due to energy efficiency and reduced consumption.

- New market opportunities for sustainable products and services.

- Meet regulatory and legal requirements.

- Include comprehensive recycling programs.

- "Paperless" business.

Minatogawa VL et al., developed an artifact to assist BMI using performance measurement indicators of a company's business model powered by big data analytics. They successfully applied and demonstrated this for a fashion e-commerce company, thereby proving that the process of BMI can be greatly enhanced by applying big data analytics [19].

Data-driven business model innovation should plan to better harness data by:

- Improving customer experiences.

- Personalising products and services to customers.

- Tracking business performance.

- Making strategic decisions through insights gained into markets, customers and clients.

- Targeted advertising.

- Improve internal and supply chain efficiencies.

- Recording behaviors and activity to track accountability and improve security.

Any business model should fundamentally refer to the way business wants to operate related to the following seven business dimensions [18].

- Value proposition - Values which a business proposes to its customers, users, employees, or supplier, which can be products, services, or processes ideally generating a profit to the business. However, this value need not be in terms of money. It could be knowledge dissemination, goodwill earned, or even happiness through contribution to humanity.

- Customers or Users - Customers pay for the value directly, whereas user does not pay directly. Customers can be end consumers (B2C) or businesses (B2B). Value is derived from users indirectly, for example, social media users. 


\section{S. K. Rao}

- Value chain functions - Activities which are required to create product, service, or the process.

- Competences - Internal competencies required to perform the value chain functions, which could be technology, human resources, organization structure, and its culture.

- Network - These are partners of the business, which could consist of physical networks like internal colleagues or supply chain partners or tax auditors, etc. ICT systems form part of the digital network. Networks could also be virtual, that is, they are not part of the business throughout, but come into picture as and when required only.

- Value formula - Procedure for calculating price and costs, which can be quantified in terms of money or other values. This indicates whether the business is making profit or loss.

- Relations - This holds all the above dimensions together by setting a relation amongt them. Such a relation could be channelized through our physical senses (seeing, hearing, feeling, tasting, and smelling) or could be virtual.

The challenges for developing a data-driven $\mathrm{BM}$ are in extracting data, refining it, and ensuring that it is utilized most effectively. Companies must first have clarity to know what to achieve by using big data. They should then formulate the value proposition, that is, plan the desired offerings and the target customers. The target customers could be businesses (B2B), Consumers(B2C), Consumers through other businesses (B2B2C), or Government (B2G). They should decide what data is required and the methods of acquiring the data. The data sources could be either self-generated, existing data, freely available data, provided by customer, or acquired data.

Next, they should decide on the method of processing this data to extract the true value contained within data, which could be done by descriptive analytics, predictive analytics, or prescriptive analytics in increasing order of sophistication depending on the level of investments in tools and resources. Further, they should decide the methods of monetizing the offerings.

The revenue model could consist of Licensing, Subscription fees, Usage fee, Product sale, Renting/leasing, Advertising, etc., depending on the offerings and the target market. The challenges faced in accomplishing the desired outcome using the data-driven BM are the quality and relevance of data and the sophistication of the analytics methods used [15].

Business models should be technology driven to ensure that future market conditions and customer needs are understood by harnessing the full 
potential of data. This would ensure business survival in a highly competitive environment [16].

Data-driven business models have two main elements: The first one is value creation, which is dependent on the availability of the right data inhouse or acquired from outside sources. Some initial test data can be used which can be further enriched via external data collection.

Next, the usage of this data must be determined with analytics, that is, to create the objective of this data. The value capture process involves planning the conversion of this data into revenue earning service by exploring the target customers. This gives a clear target market when transforming value creation into value capture.

The second element is the creation of the revenue model to be used which should make the value proposition appealing to targeted customers, thereby ensuring business sustainability. If data is not immediately available or it cannot be processed in-house, then engaging with the right partners becomes necessary.

Business Models Inc defines three categories of big data business models based on their value proposition and customers - "Data-as-a-Service Business Model," "Information-as-a-Service Business Model," and "Answers-asa-Service" [17].

“Data-as-a-Service” Business Model Category [17]

Here, the requirement for the customer/user is only aggregated and anonymized data, which they can use to develop their own offerings. This model has an easier business development process and lower revenue potential and is presented in Figure 1.

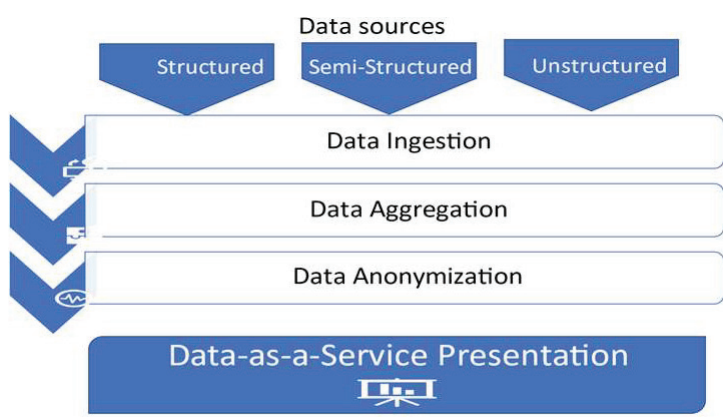

Figure 1 "Data-as-a-service" business model category. 
Describing this business model as per the "Seven dimensions of business" [18].

- Value proposition - Aggregated and anonymized data to customers who then can utilize this data in whatever way they want.

- Customers or users - Those entities who have the capability and the expertise to use raw data and derive their own information or insights from this data and gain benefits from the offerings they develop from such insights.

- Value chain functions - Data ingestion from different data sources which may include structured, semi-structured, or unstructured data, data aggregation, and data anonymization.

- Competencies - Data expertise, data collection, data management.

- Network - Could be physical, digital, or virtual network providing raw data.

- Value formula - Business intelligence/Data platforms usage-based.

- Relations - Analytics service providers, business intelligence providers, data sources, etc.

\section{“Information-as-a-Service” Business Model Category [17]}

Here, the requirement for the customer/user is some insights or information which they can use for their business or work. This business model is about converting data into information for customers who may pay for some customized information and is presented in Figure 2.

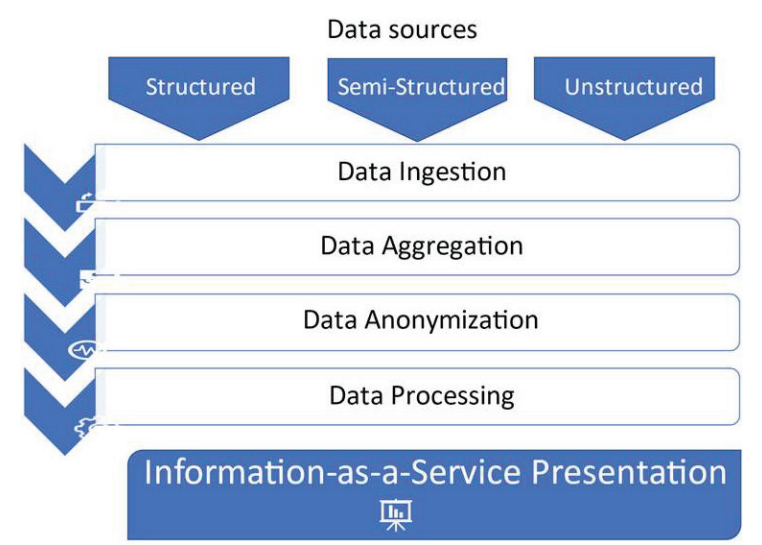

Figure 2 "Information-as-a-service" business model category. 
Describing this business model as per the "Seven dimensions of business models" [18].

- Value proposition - Aggregated, anonymized data, further processed, to extract some information/insights specifically of use to customers, who may not have the expertise for this or may not want to invest in this.

- Customers or Users - Any business or organization who could use these insights for their own use or for further selling an idea based on certain information.

- Value chain functions - Data ingestion from different data sources which may include structured, semi-structured, or unstructured data, data aggregation, data anonymization, data processing/visualization.

- Competencies - Data expertise, data collection, data management, data analytics platforms, data analysis expertise.

- Network - Data sources, big data analytics platform providers, cloud platforms, etc.

- Value formula - On-premises or SaaS model-based data platforms usage.

- Relations - Analytics/Business intelligence service providers, data sources, etc.

\section{“Answers-as-a-Service” Business Model Category [17]}

This provides an accurate answer to specific questions of the customers, who may need this for making an immediate decision. This model ensures that the answers generated are beneficial to the customer in terms of authenticity, reliability, and potential value, which can provide confidence to the customer to make spontaneous decisions with these answers and hence would be willing to pay a higher price for this. This is presented in Figure 3.

Describing this business model as per the "Seven dimensions of business models" [18].

- Value proposition - Aggregated, anonymized data, further processed to extract some information specifically of use to customers and perform advanced analytics to provide accurate answers to specific customer's requirements.

- Customers or Users - Businesses, marketing companies, on-line shopping, etc.

- Value chain functions - Data ingestion from different data sources which may include structured, semi-structured, or unstructured data, 


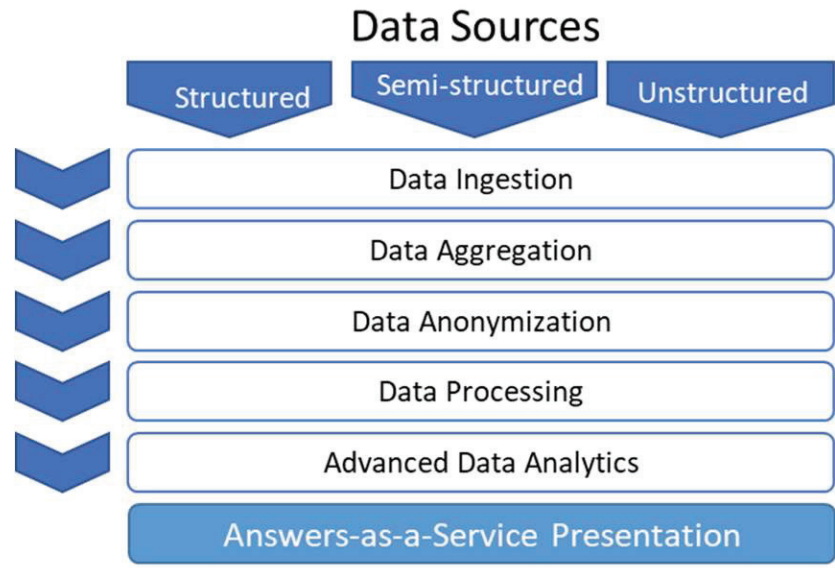

Figure 3 "Answers-as-a-service" business model category.

data aggregation, data anonymization, data visualization, data analytics, what-if scenario development, and predictive analytics.

- Competencies - Data expertise, data collection, data management, advanced data analytics platforms, data analysis expertise, customer and market knowledge.

- Network - Data sources, big data analytics platform providers, cloud platforms, etc.

- Value formula - Data analytics product sales, SaaS model-based data analytics, etc.

- Relations - Analytics/ Business intelligence service providers, data sources, etc.

\section{Intelligent Transport Systems Data-driven Business Model Innovation}

Data-driven business model innovation for intelligent transport systems, is discussed here as an example of how real-time data can be harnessed to create value. The conventional business models of vehicle manufacturers focused on selling more vehicles. They need to change their business models from product-oriented strategy to a customer- and service-oriented strategy. With new concepts of ridesharing, next-gen mobility, they should shift their business model focus away from physical vehicles to "custom design for individual" i.e., allow the customer to specify lot of convenience in the vehicle as per their preference. With personal context at the 
forefront, the driver and rider experience are becoming the true market differentiator.

The business model should provide a seamless, personal, relevant experience to the user. The data to create this experience exists in abundance, and it is only growing more plentiful. The business models should enable the system to collect this data, leverage it to infer context, and create bold, revolutionary solutions. Inherently, hardware acts as a commodity, largely static, and lacking dynamism. But using data to create a highly contextual, adaptable experience introduces a new paradigm: one where automobiles are no longer commodities but intimate, personal platforms [1].

Business models should enable the following services through vehicle data analytics which are valuable to vehicle manufacturers, vehicle users, third party service providers, and government agencies [1]:

- Usage-based insurance.

- Usage-based tolling and tax.

- Connected navigation services.

- Remote control of vehicles.

- Driver's condition monitoring service.

- Emergency call service.

- Breakdown call service.

- Stolen vehicle tracking and theft protection.

- Targeted advertisements and promotions.

- Fleet management services.

- Remote vehicle performance configuration.

- Vehicle usage monitoring and scoring.

Business models should address leveraging of data to drive more relationships and experiences between drivers and their vehicles for monetization. In a connected vehicle ecosystem, abundance of data will be created which can potentially provide insights into vehicle health and customer behavior. Business models should include services such as predictive maintenance to predict future failure of vehicle parts and direct users to service centers before parts can malfunction.

Insurance companies can offer usage-based insurance analyzing the driver's capability and usage patterns. Location-based services can be offered to drivers based on analysis of their personal preferences and behavior data.

Connected vehicle data can be used to increase internal efficiency, quality, and product differentiation for vehicle manufacturers. This data could be used to calibrate their own vehicles and redesign them according to the insights 


\section{S. K. Rao}

they got about vehicle performance. Data can be used to make smarter decisions about when to replace wear components in the vehicle instead of on regular service intervals.

Further, data can help improve vehicle health through driving statistics, self-diagnosis, remote diagnosis, and real-time vehicle information. These are things that become even more important when moving to a business model where the OEM is the owner of the vehicle.

The new business model for the vehicle manufacturer includes creating partnerships between Telcos, application providers and other third parties. Such partnerships will become important in creating value for them outside their current value chain, where they will need to package data in ways that make it attractive.

Business models could include the costs of data monetization being bundled in the vehicle price or offering a subscription price. Different players to harness vehicle data monetization include vehicle manufacturers, telecom service providers, infrastructure providers, insurance companies, third party mobility aggregators, etc.

The traditional vehicle manufacturers, and their parts' OEMs will find it challenging in adopting to this scenario. They need to make paradigm changes to monetize the full potential of vehicle data.

Examples of data-driven business model innovation implementation in urban transport: [20] - An Indian state is implementing a structured framework for data-driven systems approach to road safety with the following objectives:

- Enhance road safety measures to reduce number of road accidents and fatalities from it.

- Increase survivability of road accident victims and reduce occurrence rate.

- Capacity building of the core road safety team.

- Develop road map towards reaching the SDG goal of 50\% reduction in road fatalities by 2030 and eventually to zero deaths from road traffic accidents.

- Help build a data-driven trauma care.

\section{Conclusions}

6G technologies enable new use cases across multiple domains like Smart cities, Connected vehicles, Factories of the future, Healthcare, Security \& Surveillance, Utilities, Media \& Entertainment, etc. This will further add 
to the data explosion happening currently in the enterprise area, generating humongous amount of data which needs to be harnessed in real-time or nearreal time to create value. Further, insights from "dark data" also needs to be harnessed for competitive advantage. Organizations are unable to harness data effectively to get actionable insights due to the limitations of the traditional data analytics platforms which they use and the absence of data-driven organizational culture.

The above challenges can be addressed by (a) utilizing advanced AIbased data analytics platforms overcoming the limitations of traditional data analytics platforms, (b) implementing data-driven business model innovation.

Data-driven business model innovation is very important to harness the opportunities enabled and ensures businesses to remain competitive and sustainable. This ensures that business become "future ready."

Data-driven business model innovation ensures sustainability and pandemic resiliency by using data to get holistic view and adapt accordingly quickly. Data is key for navigating the digital economy. It is becoming more important to use a wider range of data, including insights and thought leadership compiled by industry experts.

Future-ready business harness data effectively, use it in a smarter and more strategic ways, leading to its sustainability. Such companies use data to make the best commercial decisions.

Data-driven business model innovation will differentiate from traditional businesses, in the way of revenue generation and customer relationships. Businesses should build data in at the heart of their business model ensuring security, resilience, and sustainability.

\section{References}

[1] Rao. S.K "Socio-Economic impact of 5G Technologies" Thesis 2018. Aarhus University, Denmark.

[2] Source: Data Age 2025 report sponsored by Seagate Technology.

[3] "Capturing the $\$ 47$ Billion Connected Vehicle Opportunity" Harbor Research. [http://harborresearch.com/connected-vehicle-opportunity]

[4] "Monetizing vehicle data" McKinsey \& Company 'Advanced Industries 'Report Sept 2016.

[5] J.Johnson, KPI Partners, 2013. https://www.kpipartners.com/blog/bid/1 42767/gartner-cio-survey-business-intelligence-as-top-priority-in-20 13

[6] Walker B, Big Data Infographic 'Dihuni' Apr 2020. 
[7] "The top Big Data Challenge remains the same" Gartner Survey 2015.

[8] Source: "High Stakes, High Rewards" report by Forbes Insights.

[9] "Data-driven Business Models" by NEMODE-New Economy Models in Digital Economy. http://blogs.exeter.ac.uk/nemode/research/data-dr iven-business-models/

[10] Rao S.K., Prasad R., "Impact of 5G Technologies on Smart City Implementation" 2018, Springer Wireless Personal Communications, 100(1), 161-176 DOI: 10.1007/s11277-018-5618-4

[11] Alina Sorescu "Data-driven Business Model Innovation" Jun 2017 'Journal of Product Innovation Management'.

[12] Marshall, Mueck, and Shockley, "How leading organizations use big data and analytics to innovate" 2015; "Strategy and Leadership Sep 2015.

[13] E. Brynjolfsson, L. Mitt, H. Kim, 'Strength in Numbers: How Does Data-Driven Decision-Making Affect Firm Performance? Social science research network paper.

[14] Dixon M, 18 Jan 2019 “Customer Success, Data Analytics” https://sele ritysas.com.

[15] Brownlow J et al. "Data and Analytics- Data-driven Business Models: A blueprint for Innovation". Cambridge Service Alliance; University of Cambridge Feb 2015.

[16] Martin De Saulles, "Best practices for building a data-driven business model" 17 Sep 2019. "Information Matters" https://informationmatters .net/best-practice-data-driven-business-model.

[17] Justin Lokitz- Business Models Inc. www.businessmodelsinc.com/bigdata-business-models.

[18] Lindgren P, Gade M; "Multi Business Model - Beestar and the seven dimensions" The Bee Business 2017.

[19] Minatogawa VL et al., "Operationalizing Business Model Innovation through Big Data Analytics for Sustainable organisations", 30 Dec 2019 Article in Sustainability of MDPI.

[20] http://bweducation.businessworld.in/article/Govt-Of-Rajasthan-To-Im plement-Data-driven-Systems-Approach-To-Road-Safety-In-Collabo ration-With-IIT-Madras/23-12-2020-357209/.

[21] David Reinsel, John Gantz, and John Rydning, "Data Age 2025: The Evolution of Data to Life-Critical," IDC white paper, April 2017, seagat e.com/files/www-content/our-story/trends/files/Seagate-WP-DataAge2 025-March-2017.pdf. 
[22] NewVantage, "Big Data Executive Survey 2017," New Vantage Partners LLC, newvantage.com/wp-content/uploads/2017/01/Big-Data-Executi ve-Survey-2017-Executive-Summary.pdf.

[23] Johnson, Heather (2015-10-30). "Digging up dark data: What puts IBM at the forefront of insight economy". Silicon ANGLE. Retrieved 201511-03.

[24] ETCIO "68\% of data available to businesses goes unleveraged" Report" 17 July 2020.

[25] 'Flood of Data Will Get Generated in Autonomous Cars' AutoTech Review by Suhrid Barua, July 2020.

\section{Biography}

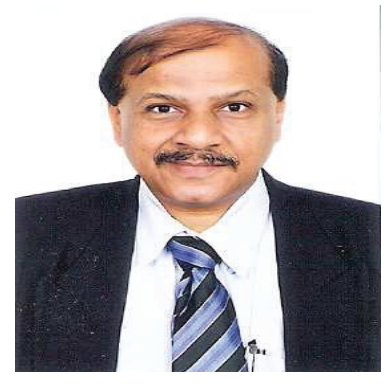

Sriganesh K. Rao is an ICT Professional with more than 35 years of global experience in leading complex technology projects with rich background in Engineering, Business development, QA \& Program Management in Telecom, Government \& Defence verticals across Embedded, Engineering Services, Testing and Manufacturing horizontals.

His experience spans across leading IT organizations like Tata Consultancy Services \& IBM, Telecom Service Providers like OPTUS (Australia) \& TATA Teleservices, Telecom OEMs like Bharat Electronics, United Telecoms \& ERG (Australia), and Telecom OSS/BSS Providers like Alopa Networks. Currently, he serves on the Board of Calligo Technologies, a Data Science and Product company at Bangalore, India.

He received his M. Tech (Electronics) degree from NITK Surathkal, MBA (Technology Management with focus on International Telecommunication Management) from Deakin University (Australia). He obtained his $\mathrm{PhD}$ in the area of Business Development and Technology related to 5G technologies from Aarhus University, Denmark. 
Sriganesh's current interests include 5G, M2M, IoT, Smart Cities, Industries 4.0, Business Model Innovation, and Big Data Analytics. He has served as the Chairman of "M2M Gateway \& Architecture" Workgroup setup by Telecommunication Engineering Centre (TEC)-Department of Telecom (DoT) of Government of India. He is a Member of the National Working Group of DoT (TEC) for IoT and its application in Smart Cities. He was also the Vice Chair of the Consultative Committee for the adoption of oneM2M Standards by TEC as National Standards.

$\mathrm{He}$ is a Senior Member of IEEE, Senior Member and Certified Professional of Australian Computer Society, Life Member of Computer Society of India. Earlier, he was the President of Australian Alumni Association, Bangalore. Currently, he is the National President of Deakin University Alumni India Chapter. 